\title{
Manejo de mediastinitis posterior a cirugía cardiaca: reporte de caso
}

\author{
Diego Humberto Márquez-Bohórquez* \\ Jaime Calderón-Herrera**
}

\begin{abstract}
*Médico. Especialista en Cirugía General. Universidad Industrial de Santander. Bucaramanga, Colombia.
**Médico. Especialista en Cirugía General y Cirugía Cardiovascular. Instituto del Corazón de Bucaramanga. Profesor Cirugía General. Universidad Industrial de Santander. Bucaramanga. Colombia.

Correspondencia: Dr. Diego Humberto Márquez B. Dirección: Carrera 28 \# 47-47. Apartamento 503. Edificio Domus Aurea. Bucaramanga. Santander. Colombia.Correo electrónico: diegohmarquezb@gmail.com
\end{abstract}

\section{RESUMEN}

La mediastinitis es una complicación rara pero devastadora en cirugía cardíaca, la cual incrementa los costos en salud. Los pacientes con mediastinitis posterior a cirugía cardíaca tienen alto índice de morbilidad y mortalidad. La obesidad es el único factor de riesgo perioperatorio que es modificable. El objetivo de éste reporte de caso es describir el manejo de la mediastinitis posterior a cirugía cardíaca que incluye un sistema de cierre asistido por presión de vacío con posterior fijación de la pared torácica a través de barras de titanio. MÉD. UIS. 2017;30(1):107-111.

Palabras clave: Mediastinitis. Cirugía Torácica. Terapia de Presión Negativa para Heridas. Titanio.

\section{Management of mediastinitis after cardiac surgery: a case report}

\section{ABSTRACT}

Mediastinitis is a rare and devastating complication of cardiac surgery, it increases the cost of care. Patients with mediastinitis after cardiac surgery have higher morbidity and mortality. Obesity is the only modifiable perioperative risk factor for mediastinitis. The aim of this case report is to describe the management of mediastinitis after cardiac surgery that includes vacuum assisted pressure with a subsequent fixation of the thorax wall with titanium bars. MÉD.UIS. 2017;30(1):107-111.

Keywords: Mediastinitis.Thoracic Surgery. Negative-Pressure Wound Therapy. Titanium.

¿Cómo citar este artículo?: Márquez-Bohórquez DH, Calderón-Herrera J. Manejo de mediastinitis posterior a cirugía cardíaca: reporte de caso. MÉD.UIS. 2017;30(1):107-111.

\section{INTRODUCCIÓN}

La mediastinitis posterior a cirugía cardíaca tiene una prevalencia que va desde el uno al dos por ciento $^{1-7}$. Adicionalmente, en cirugía coronaria incrementa los costos en los hospitales hasta el $240 \%$ comparado con los procedimientos no complicados ${ }^{2}$. La incidencia usual en caso de reemplazo valvular ronda el uno por ciento y con derivación coronaria, el 1,5\%. En los trasplantes la incidencia de mediastinitis puede alcanzar el cinco por ciento y superar el 50\% en caso de asistencia circulatoria central ${ }^{4}$. El objetivo de éste reporte de caso es describir el manejo de la mediastinitis en un paciente sometido a cirugía cardíaca de revascularización coronaria y recambio valvular aórtico, donde asociado al tratamiento antibiótico guiado por cultivos, se utilizó una terapia de cierre con presión negativa y posteriormente se fijó la pared torácica con barras de titanio, permitiendo restaurar la fisiología de la mecánica ventilatoria. 


\section{FACTORES DE RIESGO}

Los factores de riesgo prequirúrgico incluyen obesidad, diabetes, Enfermedad Pulmonar Obstructiva Crónica (EPOC) y la edad avanzada ${ }^{4,7}$. Adicionalmente, son factores de riesgo el afeitado en vez de depilación, la profilaxis antibiótica inadecuada, el tiempo prolongado de circulación extracorpórea y el uso de una arteria mamaria interna o las dos. El riesgo de mediastinitis se produce con la devascularización esternal subsecuente al uso de las arterias mamarias internas, llevando a isquemia, necrosis y dehiscencia ósea, especialmente cuando se hace uso excesivo del electrobisturí y la cera hemostática4. Dentro de los factores de riesgo postoperatorio se encuentra la transfusión masiva de hemoderivados ${ }^{1}$.

\section{FISIOPATOLOGíA}

Existen diferentes mecanismos fisiopatológicos que Ilevan a mediastinitis. El primero de ellos consiste en un compromiso de la integridad esternal que permite la migración de gérmenes cutáneos hacia el mediastino, seguido por la infección esternal primaria. La contaminación es intraoperatoria en la mayoría de los casos y ocurre a partir de la flora bacteriana del paciente. También se puede originar de una bacteremia originada en focos infecciosos extra-torácicos, que podría explicar algunos casos de mediastinitis por bacilos gramnegativos 4 .

\section{Microbiología}

Los microorganismos más frecuentes son los cocos gram positivos, teniendo como referente el $S$. aureus y los estafilococos coagulasa negativos. Las bacterias gramnegativas representan del 20\% al 40\% de los microorganismos patógenos ${ }^{3}$, que incluyen enterobacterias, Pseudomonas, Acinetobacter $y$ Citrobacter $^{4}$. La mediastinitis secundaria a bacterias gramnegativas está asociada a una disminución en la sobrevida, en muchas ocasiones debido a un inapropiado esquema antibiótico ${ }^{3}$. En estos casos, la diseminación hematógena prevalece más que la inoculación directa.

\section{Diagnóstico}

El diagnóstico es clínico, al observar signos como fiebre, drenaje de material purulento en la herida quirúrgica, dolor e inestabilidad esternal, lo cual se hace evidente al palpar los bordes óseos. Posteriormente, se debe confirmar la sospecha clínica con el aislamiento del microorganismo a través de los cultivos de muestras. Si alguno de éstos es informado negativo, pero existe alta sospecha clínica de mediastinitis, no se debe descartar el diagnóstico y se deben tomar nuevas muestras ${ }^{4}$.

\section{TRATAMIENTO}

La profilaxis antibiótica debe incluir estafilococos. Se recomienda una cefalosporina de primera o segunda generación, generalmente cefazolina, la cual se debe administrar una hora antes de la incisión y reforzarse si el procedimiento quirúrgico es prolongado. El manejo antibiótico empírico también debe cubrir estafilococos, teniendo en cuenta la incidencia de cepas meticilino resistentes. Se recomienda vancomicina, un aminoglucósido y cefotaxime, posteriormente se puede reemplazar el aminoglucósido por un antibiótico con buena absorción ósea como una quinolona o la rifampicina ${ }^{4}$. La duración total del tratamiento antibiótico es de seis semanas y se debe ajustar según el informe de los estudios microbiológicos. El manejo quirúrgico incluye las técnicas a tórax abierto donde el esternón y la piel se dejan abiertos.

\section{Presentación del Caso}

Paciente de 76 años, masculino, con antecedente de hipertensión arterial, EPOC no exacerbada e índice de masa corporal en 35; quien presenta dolor torácico y disnea de larga evolución, asociada al esfuerzo físico y que ocurre ocasionalmente en reposo. Fue estudiado documentando estenosis aórtica severa y enfermedad coronaria multivaso. Dentro de la valoración prequirúrgica no se encontró contraindicación para el procedimiento quirúrgico, evidenciando valores normales de hemoglobina (14 $\mathrm{g} / \mathrm{dL}$ ), plaquetas (250 000 por $\mathrm{mcL}$ ) y creatinina $(0,9$ $\mathrm{mg} / \mathrm{dL})$. Se consideró indicado manejo quirúrgico de la patología valvular e isquémica coronaria. Se realizó esternotomía, resección de la válvula nativa e implante de bioprótesis más revascularización miocárdica con anastomosis de la arteria mamaria izquierda a la arteria descendente anterior y puentes de vena safena a las arterias coronarias derecha y obtusa marginal, sin complicaciones en el transoperatorio. 
Posteriormente, en su estancia en la Unidad de Cuidado Intensivo el paciente presentó picos febriles, drenaje de material purulento a través de la herida quirúrgica e inestabilidad esternal. El paciente se encontraba con soporte ventilatorio invasivo, estable hemodinámicamente sin soporte inotrópico ni vasopresor. Se realizó manejo con tórax abierto y sistema de cierre asistido por presión de vacío (VAC, de sus siglas en inglés) (Ver Figura 1), cubrimiento antibiótico empírico con piperacilina, tazobactam y vancomicina; ajustado por el cultivo de secreción mediastinal que informó Pseudomonas sensible a piperacilina tazobactam, suspendiendo la vancomicina y completando ocho semanas de tratamiento.

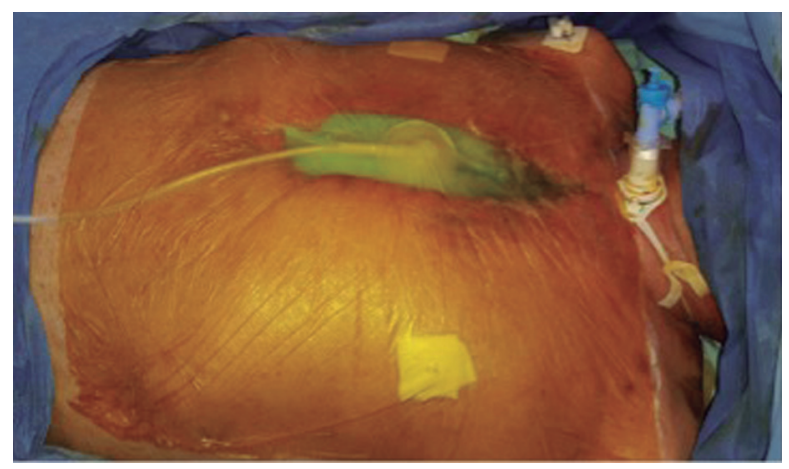

Figura 1. Cierre asistido por presión de vacío (VAC).

Fuente: autores

Como factores de riesgo prequirúrgico para mediastinitis el paciente presentaba EPOC y obesidad, los factores de riesgo en el transoperatorio incluyen el uso de la arteria mamaria interna para la revascularización miocárdica. En el posoperatorio el paciente requirió traqueostomía por intubación orotraqueal prolongada, lo cual representó un factor de riesgo para mediastinitis dado que la herida quirúrgica de esternotomía debía ser protegida estrictamente para que no estuviera en contacto con las secreciones traqueales. Los cambios de la terapia VAC se realizaron cada 72 horas durante las primeras cuatro semanas postoperatorias dado el alto riesgo de contaminación de la herida quirúrgica con las secreciones traqueales. Se realizó cierre gradual de la piel proximal en la incisión de esternotomía para separar la cánula de traqueostomía de la herida quirúrgica. Después del primer mes, los cambios de la terapia VAC se hicieron cada cuatro a cinco días dado que la secreción disminuyó el volumen y era serohemática. El paciente requirió 12 recambios de la terapia VAC durante los dos meses de estancia hospitalaria. Finalmente, después de tener controlado el foco infeccioso con informe de cultivos negativos y contar con adecuado tejido de granulación, se realizó cierre y fijación de la pared torácica con barras de titanio (Ver Figura 2).

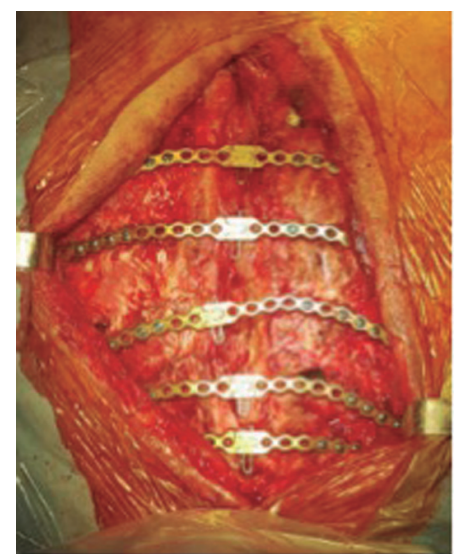

Figura 2. Cierre y fijación de pared torácica con barras de titanio. Fuente: autores

En la evolución postoperatoria el paciente requirió manejo con analgesia formulada por la clínica de dolor y terapia respiratoria estricta. Su estancia en Unidad de Cuidados Intensivos posterior al cierre esternal fue de tres días. La decanulación traqueal fue exitosa en la primera semana después de la última intervención y el egreso hospitalario a los 18 días. El control en consulta externa a los diez y 30 días del alta evidenció un paciente con mecánica ventilatoria adecuada, adecuado estado nutricional, sin signos de infección en las heridas quirúrgicas, sin disnea ni nuevos episodios de angina ni dolor torácico.

\section{Discusión}

La esternotomía mediana es el procedimiento estandarizado para realizar cirugía cardíaca. Sin embargo, la mediastinitis posterior a esternotomía es una complicación devastadora, especialmente cuando se realiza revascularización miocárdica. Muchos estudios han demostrado un incremento en la morbilidad y mortalidad, así como disminución en la sobrevida a largo plazo, con la mediastinitis posterior a la realización de puentes coronarios ${ }^{4}$. A pesar que la incidencia de la mediastinitis post-esternotomía es baja, la cual oscila entre $0,5 \%-5 \%$, ésta tiene una alta mortalidad (20\%-33\%). Serap et al., estudió 19767 pacientes que fueron llevados a cirugía cardíaca, de los cuales 117 (0,39\%) presentaron mediastinitis post-esternotomía, 32\% de estos 117 pacientes fallecieron. Grauhan et al., estudió 150 pacientes obesos que fueron llevados a esternotomía mediana encontrando un riesgo de 6,1\% para desarrollar mediastinitis post-esternotomía13,14. 
La técnica VAC fue descrita inicialmente por Argenta y Morykwas. Los resultados de los estudios demuestran que la terapia VAC es una herramienta útil, posterior al debridamiento exhaustivo, para el manejo de la mediastinitis post-esternotomía. La terapia VAC consiste en aplicar presión negativa al tejido para sellar la herida, la fuerza de succión creada permite el drenaje de fluidos excesivos y el debridamiento tisular, Ilevando a la remoción del edema y la reducción en el recuento bacteriano promoviendo la proliferación celular, el crecimiento de tejido de granulación y el cierre de la herida. Diferentes estudios han demostrado que la terapia VAC incrementa el diámetro capilar y la velocidad del flujo sanguíneo, además promueve la angiogénesis y la proliferación celular endotelial ${ }^{8}$. Chen et al., en el año 2005 estudió 32 ratas blancas japonesas de orejas largas a las cuales les realizó una herida de dos centimetros de diámetro y de espesor total en la oreja derecha e izquierda. La herida de la oreja izquierda fue recubierta por una espuma estéril, a la cual se le aplicaba una presión negativa de -5, -10, -15 y -20 kPa, cada una duraba 20 minutos. La herida en la oreja derecha fue recubierta por gasa vaselinada. En el transcurso del experimento se realizaron imágenes con un microscopio de microcirculación. Chen et al., encontró que en la herida de la oreja izquierda el sistema de presión negativa promovió mayor velocidad de flujo sanguíneo capilar, incrementó el diámetro de los capilares y el volumen sanguíneo, estimuló la proliferación endotelial y la angiogénesis, acortó los espacios endoteliales y restauró la integridad de la membrana basal de los capilares ${ }^{15}$.

Cuando el defecto esternal no es muy grande, la terapia VAC es suficiente para el manejo de la mediastinitis postesternotomía. Existen estudios que han reportado efectividad en un abordaje simplificado para los defectos pequeños. Merrill et al., en el año 2004 demostró una efectividad de $95 \%$ con éste tratamiento. Este abordaje consiste en debridamiento de la herida, cierre sobre drenes e irrigación mediastinal anterior ${ }^{9}$. Sin embargo, en los pacientes con heridas de gran tamaño, es decir, con compromiso severo de la superficie esternal que no permita su afrontamiento con puntos de alambre, el cierre sobre drenes no es posible y la terapia VAC no permite generar un tejido firme que estabilice la pared torácica, Ilevando a una mecánica ventilatoria defectuosa. La terapia VAC promueve el crecimiento de tejido de granulación, rico en fibroblastos, capilares y colágeno, que no es lo suficientemente firme para soportar las presiones de la cavidad torácica, requiriendo el cierre óseo para restaurar la mecánica ventilatoria. La otras limitaciones de la terapia VAC incluyen su alto costo y el riesgo de lesionar los puentes venosos de la revascularización miocárdica dada la exposición directa de la espuma con el mediastino.

En estos casos, posterior al manejo con terapia VAC, se puede utilizar un colgajo de músculo pectoral mayor y recto abdominal con doble pedículo como método práctico y eficaz en la reconstrucción del defecto en la pared anterior del tórax causado por la mediastinitis post-esternotomía. Éste colgajo no solamente provee suficiente volumen para cubrir el mediastino en su totalidad, sino que además permite la resolución del cuadro infeccioso con resultados favorables ${ }^{10}$.

Este reporte de caso describe el uso de la terapia VAC con posterior fijación de la pared torácica a través de barras de titanio, permitiendo el cierre de un defecto esternal importante y la restauración temprana de la mecánica ventilatoria, sin necesidad de recurrir a colgajos musculares, obteniendo resultados favorables y sin complicaciones. En el año 2014, Tarzia et al. recolectaron retrospectivamente 7148 pacientes que fueron llevados a cirugía cardíaca, encontrando que 152 (2,1\%) presentaron dehiscencia de la herida de esternón, de los cuales 107 fueron tratados con medidas convencionales y 45 con terapia VAC. La mortalidad, incidencia de mediastinitis, sepsis y revisión quirúrgica esternal fue menor en el grupo de terapia VAC ${ }^{11}$. En el año 2014, Risnes et al. demostraron que los pacientes que son llevados a revascularización miocárdica con puentes coronarios y presentan mediastinitis, tienen una mayor tasa de re-infección y falla en el cierre esternal cuando son tratados con medidas convencionales comparado con los que se manejan con terapia VAC ${ }^{12}$.

Este trabajo presenta las limitaciones propias de todo reporte de caso.

\section{Conclusiones}

La mediastinitis es una complicación grave de la cirugía cardíaca, la cual debe diagnosticarse clínicamente y tratarse de manera temprana con antibiótico sistémico guiado por los informes de cultivos. Sin embargo, cuando el proceso infeccioso es muy avanzado y se ha comprometido gran parte del tejido esternal, éste manejo no es suficiente. En 
estos casos, se expone un tratamiento quirúrgico adicional con VAC y posteriormente fijación de la pared torácica con barras de titanio, una vez los cultivos de secreción sean negativos, permitiendo restaurar la fisiología de la mecánica ventilatoria.

\section{Consideraciones Éticas}

Se contó con la autorización de manera voluntaria del paciente para la divulgación de la información consignada.

\section{RefERenCias Bibliográficas}

1. Ang LB, Veloria EN, Evanina EY, Smaldone A. Mediastinitis and blood transfusion in cardiac surgery: a systemic review. Heart and Lung. 2012;41(3):255-63.

2. Atkins BZ, Onaitis MW, Hutcheson K a., Kaye K, Petersen RP, Wolfe WG. Does method of sternal repair influence longterm outcome of postoperative mediastinitis? Am J Surg. 2011;202(5):565-7.

3. Charbonneau H, Maillet JM, Faron M, Mangin O, Puymirat E, Le Besnerais P, et al. Mediastinitis due to Gram-negative bacteria is associated with increased mortality. Clin Microbiol Infect. European Society of Clinical Infectious Diseases. 2013;20(3):197202.

4. Guérot E. Mediastinitis después de cirugía cardíaca. EMCAnestesia-Reanimación.2013;39(3):1-8.

5. Lepelletier D, Poupelin L, Corvec S, Bourigault C, Bizouarn P Blanloeil Y. Risk factors for mortality in patients with mediastinitis after cardiac surgery. Arch Cardiovasc Dis. 2009;102(2):119-25.

6. Mekontso Dessap, Vivier E, Girou E, Brun-Buisson C, Kirsch M. Effect of time to onset on clinical features and prognosis of post-sternotomy mediastinitis. Clin Microbiol Infect. European Society of Clinical Infectious Diseases. 2011;17(2):292-9.

7. Rehman SM, Elzain O, Mitchell J, Shine B, Bowler ICJW, Sayeed R, et al. Risk factors for mediastinitis following cardiac surgery: the importance of managing obesity. J Hosp Infect. 2014;88(2):96-102.

8. Ennker IC, Pietrowski D, Vohringer L, Kojcici B, Albert a, Vogt PM, et al. Surgical debridement, vacuum therapy and pectoralis plasty in poststernotomy mediastinitis. J Plast Reconstr Aesthetic Surg. 2009; 62(11):1479-83.

9. Merrill WH, Akhter S a, Wolf RK, Schneeberger EW, Flege JB. Simplified treatment of postoperative mediastinitis. Ann Thorac Surg. 2004;78(2):608-12.

10. Roh TS, Lee WJ, Lew DH, Tark KC. Pectoralis major-rectus abdominis bipedicled muscle flap in the treatment of poststernotomy mediastinitis. J Thoracic Cardiovasc Surg. 2008;136:618-22.

11. Tarzia V, Carrozzini M, Bortolussi G, Buratto E, Bejko J. Impact of vacuum-assisted closure therapy on outcomes of sternal wound dehiscence. Interact Cardiovasc Thorac Surg. 2014;19:70-5.

12. Risnes I, Abdelnoor M, Veel T, Svennevig JL, Lundblad R, Rynning SE. Mediastinitis after coronary artery bypass grafting: The effect of vacuum-assisted closure versus traditional closed drainage on survival and reinfection rate. Int Wound J. 2014;11(2):177-82

13. Dohmen P, Markou T, Ingemansson R, Rotering H, Hartman J, Valen R, et al. Can post-sternotomy mediastinitis be prevented by a closed incision management system? GMS Hyg Infect Control. 2014;9(3):Doc 19.

14. Yavuz S, Sensoy A, Ceken S, Deniz D, Yekeler I. MethicillinResistant Staphylococcus aureus infection: an independent risk factor for mortality in patients with poststernotomy mediastinitis. Med Princ Pract. 2014;23:517-523.

15. Chen SZ, Li J, Li XY, Xu LS. Effects of vacuum-assisted closure on wound microcirculation: an experimental study. Asian J Surg. 2005;28(3):211-7. 